\title{
Electromagnetic properties of neutrinos from scattering on bound electrons in atom
}

\author{
Junu Jeong ${ }^{(1)}$, Jihn E. Kim ${ }^{(2)}$, and Sungwoo Youn ${ }^{(1)}$ \\ (1) Center for Axion and Precision Physics Research, IBS, Daejeon 34051, Republic of Korea \\ ${ }^{(2)}$ Department of Physics, Kyung Hee University, 26 Gyungheedaero, \\ Dongdaemun-Gu, Seoul 02447, Republic of Korea
}

\begin{abstract}
We consider the effects of bound atomic electrons scattered by solar neutrinos due to the electromagnetic properties of neutrinos. This necessiate considering the recoil of atomic nucleus, which should be considered in the momentum conservation, but that effect to the energy conservation is negligible. This effect changes the kinematic behavior of the scattered electron compared to that scattered on free electrons. We apply this effect to the recent XENON1T data, but the bounds obtained from this is not very restrictive. We obtained the bounds: the (transition) magnetic moment $\left|f_{\alpha \beta}\right| \leq 0.86 \times 10^{-7}$ (times the electron Bohr magneton) and the charge radius $|\tilde{r}|<4.30 \times 10^{-17} \mathrm{~cm}$. For a non-vanishing millicharge $(\varepsilon)$, the allowed bound is shown in the $\tilde{r}^{2}-\varepsilon$ plane.
\end{abstract}

PACS numbers: 13.15.+g, 12.15.Mm, 13.40.Gp, 13.66.-a

Keywords: Neutrino Charge Radius, Neutrino Magnetic Moment, Millicharge, XENON1T

\section{INTRODUCTION}

Ever since the discovery of neutrinos, it has been a great challenge to find the electromagnetic(EM) properties of neutrinos [1 3, which continued in the gauge theory era 448 . To have the EM properties of neutrinos at the observable level, particles in the beyond-the-standard-model(BSM) had to be introduced 9-12. Two relevant EM properties of neutrinos are the magnetic moment(MM) and the charge radius $(\mathrm{CR})$. The MM, coupling to the EM field strength, is gauge invariant. On the other hand, the CR, coupling to the EM field itself, is not gauge invariant. Gauge invariance is needed to maintain the renormalizability. Therefore, if the probing wave length in the effective theory is much larger than the cutoff length-scale in the renormalization, as in the Fermi weak interaction much above the electroweak length-scale $10^{-16} \mathrm{~cm}, \mathrm{CR}$ can be also considered as a useful physical parameter. The cutoff energy scale for the CR is the mass of a heavy BSM particle whose exchange produces the relevant charge radius. In this paper, we take this viewpoint considering the electromagnetic properties of neutrinos.

The published experimental limit on the MM of $\nu_{e}$ in units of the electron Bohr magneton is $\left[13,|f| \lesssim 2.8 \times 10^{-11}\right.$, and the limit on the squared $\mathrm{CR}, \tilde{r}^{2}$ is [14], $\tilde{r}^{2}=[-2.1,+3.3] \times 10^{-32} \mathrm{~cm}^{2}$. The standard model(SM) prediction [15] on the neutrino MM is much smaller than the upper limit presented above, by a factor of $\mathrm{O}\left(10^{-8}\right)$. Note that the millicharge limit of neutron is $\mathrm{O}\left(10^{-21} \mathrm{~cm}\right)$ [16], which however cannot be directly used for a limit on the millicharges of neutrinos.

Recently, the XENON group considered the possibility of MMs of solar neutrinos for the excess events in their data, for an exposure of 0.65 tonne-year with an extremely low background rate of $76 \pm 2$ (stat.) (Events) $/(\mathrm{t} \cdot \mathrm{y} \cdot \mathrm{keV})$ 17. The plot on these excess events starts around the electron recoil energy near $2-3 \mathrm{keV}$, and ends around $30 \mathrm{keV}$ as shown in Fig. 1 (a). Out of the fitted 42,179.4 events, the estimated SM background by the solar neutrinos is 220.8 events [17, only $0.52 \%$. For estimating non-vanishing electromagnetic properties of solar neutrinos, therefore, one may neglect the SM background and associate the bulk of 42,179.4 events with the electromagnetic properties of neutrinos in the scattering process. In this way, Ref. 17] obtained the bound on the neutrino MM, $[1.4,2.9] \times 10^{-11}$ times the electron Bohr magneton. But, the assumed cross section by the XENON group has the reciprocal dependence on the recoil electron energy. This reciprocal dependence is obtained by assuming the two-body scattering [19], $\nu_{e}+e$, which may not be a correct method. The reason is that in kicking out an electron from a heavy atom, the atomic nucleus can carry away some momentum and the momentum conservation used in the $\nu_{e}+e_{\mathrm{in} \text { atom }} \rightarrow \nu_{e}^{\prime}+e^{\prime}$ is not exact.

In Sec. II we express the XENON1T unit "Events $/ \mathrm{t} \cdot \mathrm{y} \cdot \mathrm{keV}$ " in terms of the cross section unit "MeV" to compare with the theoretical prediction. In Sec. III, we discuss the possibility of including electromagnetic properties of neutrinos in the scattering process. Here, the three-body phase space with a non-relativistic atom is discussed. In Subsec. IIID, we briefly comment on the possibility of neutrino milli-charges. In Sec. IV] from XENON1T data we obtain the $\mathrm{CR}$ and millicharge bounds of electron-type neutrino. Section $\mathrm{V}$ is a brief summary on the EM properties of neutrinos. In Appendix A, we estimate the parameters used in the calculation. 


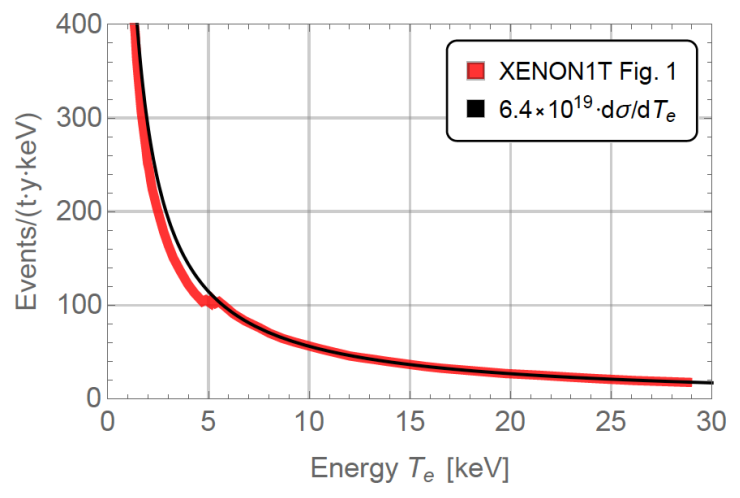

(a)

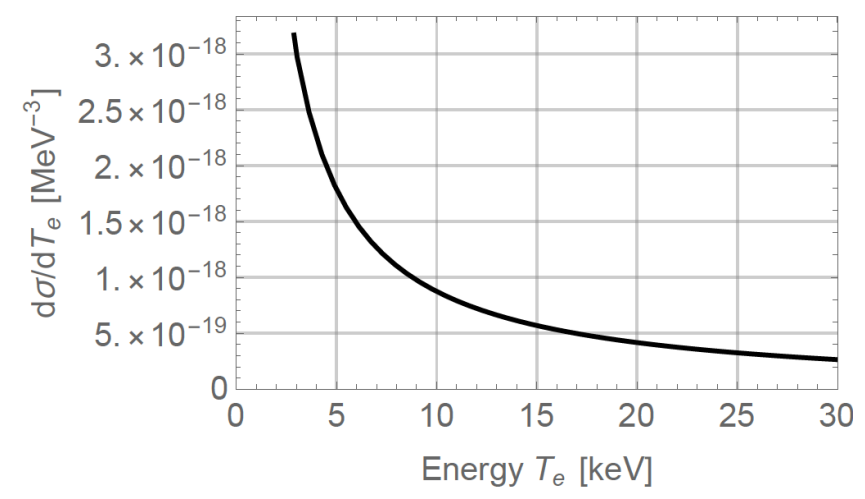

(b)

FIG. 1: (a) Cross section vs. the data curve for $\mu_{\nu}=7 \times 10^{-11} \mu_{\mathrm{B}}$ reproducing the RHS panel of Fig. 1 of [17], and (b) a conversion of the event rate to the cross section.

\section{EVENT RATE AND CROSS SECTION IN THE XENON1T DATA}

The XENON group presents data in units of Events $/ \mathrm{t} \cdot \mathrm{y} \cdot \mathrm{keV}$. To convert it to cross section, we use the right-hand side (RHS) panel of Fig. 1 of [17, and followed their analysis using the neutrino magnetic moment $\mu_{\nu}=7 \times 10^{-11} \mu_{\mathrm{B}}$ and the solar $p p$ neutrino flux of [18]. They used the inverse- $T_{e}$ rule presented in [19]. In our paper here, the $p p$ neutrino energy flux of [18] in the region, $E_{\nu} \geq 4 \mathrm{MeV}$, is fitted by $F_{\mathrm{pp}}^{\text {solar }}(E)$. The analytic form for the $p p$-flux function is presented in [33],

$$
F_{p p}^{\text {solar }}(E)=P_{0} E^{2}\left(Q+m_{e}-E\right) \sqrt{\left(Q+m_{e}-E\right)^{2}-m_{e}^{2}}
$$

where $Q \approx 423.41 \mathrm{keV}$ is the end point energy of neutrino, and $m_{e} \approx 0.511 \mathrm{MeV}$ is the electron mass. $P_{0}=$ $188.143 \mathrm{MeV}^{-5}$ is the normalization constant. From this form, some averages of energy functions are

$$
\begin{gathered}
\left\langle E^{-2}\right\rangle=30.0256 \mathrm{MeV}^{-2}, \\
\left\langle E^{-1}\right\rangle=4.46821 \mathrm{MeV}^{-1}, \\
\left\langle E^{1}\right\rangle=0.267946 \mathrm{MeV}^{1} \\
\left\langle E^{2}\right\rangle=0.0794926 \mathrm{MeV}^{2} \\
\left\langle E^{3}\right\rangle=0.0251769 \mathrm{MeV}^{3}, \\
\left\langle\ln E / Q_{\text {min }}\right\rangle=-0.528546 .
\end{gathered}
$$

Figure 1(a) is the overlap of the cross section and the RHS panel data of Fig. 1 of Ref. 17. Here, we used $Q_{\min }$ as the minimum of $\left|\mathbf{p}_{e}^{\prime}\right|^{2} /\left(2 m_{e}\right)$. Figure 1(b) is the cross section $d \sigma / d T_{e}$ in the $\mathrm{MeV}^{-3}$ units, obtained from the XENON1T data using the inverse- $T_{e}$ rule. Then, our calculation of $d \sigma / d T_{e}$ resulting from some electromagnetic properties of neutrinos, not using the inverse- $T_{e}$ rule but using our formula, will be compared with Fig. 1(b).

\section{CROSS SECTIONS WITH ELECTROMAGNETIC FORM FACTORS OF NEUTRINO}

The photon vertex does not change the chirality. For the mass and MM, therefore, a chirality change must take place. So, the MM can arise by attaching a photon vertex in the mass generating diagrams. For the CR, it is even simpler because the CR has the same chiral property as that given by the EM coupling. For the neutrino, the gauge coupling is expressed as the form factor $F_{1}^{\nu}\left(q^{2}\right)$ with $F_{1}^{\nu}(0)=0$. The CR is defined by the first term in the expansion of $F_{1}^{\nu}\left(q^{2}\right)$ in terms of momentum transfer $q^{2}$. As noted in the beginning, we use the charge radius for the scale $q^{2} \ll(\text { cutoff scale })^{2}$. In addition, if a (almost) massless extra photon (ex-photon) in the BSM sector is present, there is a possibility that neutrinos can carry millicharges [20, 21]. The chiral property due to millicharges is the same as that due to the charge radius. The vector couplings of the charge radii and millicharges of neutrinos can mix with the $G_{F}$-order SM couplings, but the interference between these vector and axial-vector interactions and the SM amplitude has not played an important role because of the tiny contribution in the data of XENON1T if it were from solar neutrinos. 


\section{A. SM cross section and beyond}

The effective interaction in the SM for the electron-type neutrino scattering on an electron is given by the interaction [22, 23],

$$
\frac{G_{F}}{\sqrt{2}} \bar{\nu}_{e} \gamma^{\mu}\left(1+\gamma_{5}\right) \nu_{e} \bar{e} \gamma_{\mu}\left(g_{V}+g_{A} \gamma_{5}\right) e .
$$

When we consider the neutrino scattering, kicking out an electron from an atom with atomic number $Z$, the following six-fermion interaction with parameter $M_{\text {eff }}$ can be considered,

$$
\frac{1}{M_{\mathrm{eff}}^{3}} \cdot \frac{G_{F}}{\sqrt{2}} \bar{\nu}_{e} \gamma^{\mu}\left(1+\gamma_{5}\right) \nu_{e} \bar{e} \gamma_{\mu}\left(g_{V}+g_{A} \gamma_{5}\right) e \bar{A} A
$$

where $A$ is treated as a quantum field with $Z-1$ atomic electrons. ${ }^{1}$ A strategy to estimate $M_{\text {eff }}$ for four-fermion interaction is given in Appendix A. We interpret $M_{\text {eff }}$ as representing the electromagnetic process occurring in the atomic orbits. For four-fermion interaction, it is a kind of definition for the atomic process. For six-fermion interaction of (4), we use the same $M_{\text {eff }}$ determined in Appendix A.

In the SM, we have

$$
g_{V}=\frac{1}{2}+2 \sin ^{2} \theta_{W}, g_{A}=\frac{1}{2}
$$

where $\sin ^{2} \theta_{W} \simeq 0.231\left[22\right.$, 24] is the weak mixing angle, and $e^{2}=g^{2} \sin ^{2} \theta_{W}$. The EM couplings of neutrinos are

$$
\bar{\nu}_{\beta} \gamma^{\mu} e F_{1 \alpha \beta}^{\nu} \nu_{\alpha} A_{\mu}^{\mathrm{em}}+\bar{\nu}_{\beta} \sigma^{\mu \nu} \frac{e f_{\alpha \beta}}{2 M} \nu_{\alpha}\left(q_{\mu} A_{\nu}^{\mathrm{em}}-q_{\nu} A_{\mu}^{\mathrm{em}}\right)
$$

where $F_{1 \alpha \beta}$ and $\frac{f_{\alpha \beta}}{2 M}$ are matrices between weak eigenstates of neutrinos, $\nu_{\beta}\left(k_{\nu}^{\prime}\right)$ and $\nu_{\alpha}\left(k_{\nu}\right)$. $f_{\alpha \beta}$ is the MM of neutrino in units of the Bohr magneton of the mass $M$ particle. For $\alpha=\beta$, Eqs. (3) and (6) combine, and in particular for the electron-type neutrino $\alpha=\beta$ we have

$$
\frac{G_{F}}{\sqrt{2}} \bar{\nu}_{e} \gamma^{\mu}\left(1+\gamma_{5}\right) \nu_{e} \bar{e}\left(\left[g_{V}+\frac{F_{1}^{\nu_{e}}}{\sqrt{2} G_{F} q^{2}}\right] \gamma_{\mu}+g_{A} \gamma_{\mu} \gamma_{5}\right) e .
$$

The SM neutrino $\nu_{\alpha}$ is a two-component spinor. Therefore, in the cross section calculation the spin sums for a Majorana neutrino is $\frac{1}{2}$ of that of a Dirac neutrino, which will be taken into account.

For calculating scattering cross section on electrons bound in the orbits of a rest nucleus, the wave function of electrons in the electron cloud around the nucleus must be considered. Let the uncertainty of the outgoing electron position be $\Delta r$. Then, the uncertainty of the momentum is $\Delta p \approx 1 / \Delta r$. We consider the recoil energy greater than 2.5 $\mathrm{keV}$ (glimpsing Fig. 1 (a) ), corresponding to to $\Delta r<3.9 \times 10^{-9} \mathrm{~cm}$ with $\Delta p=\sqrt{2 m_{e} T_{e}}$ for the electron recoil energy $T_{e}$. But, in the neutrino scattering with the incoming solar neutrino energy less than $0.45 \mathrm{MeV}$ [18], the uncertainty of neutrino position is less than $4.38 \times 10^{-11} \mathrm{~cm}$ which is much shorter than the orbit radius of the $n$-th level of Xenon, $10^{-10} n \mathrm{~cm}$. So, in the neutrino scattering we can safely use the two-body scattering formula as described in Appendix A.

The $3 \rightarrow n$ transition rate is

$$
\text { Rate }=(2 \pi)^{4-3 n} \frac{d^{3} \mathbf{p}_{1}^{\prime} \cdots d^{3} \mathbf{p}_{n}^{\prime}}{2 E_{1} 2 E_{2} 2 E_{3} 2 E_{1}^{\prime} 2 E_{2}^{\prime} \cdots 2 E_{n}^{\prime}} \frac{\delta^{4}\left(p_{1}+p_{2}+p_{3}-p_{1}^{\prime} \cdots-p_{n}^{\prime}\right)}{V^{2}}\left|\left\langle p_{1}^{\prime} \cdots p_{n}^{\prime}|T| p_{1} p_{2} p_{3}\right\rangle\right|^{2}
$$

from which we calculate the three body scattering cross section. A flux of incoming particles 1 (neutrino in our case) scatter on two particles 2 (electron in our case) and 3 (atom in our case). Thus, the cross section consists of two parts, one with a flux factor $1 /\left|\mathbf{v}_{1}-\mathbf{v}_{2}\right|$ and the other $1 /\left|\mathbf{v}_{1}-\mathbf{v}_{3}\right|$. Taking $\mathbf{v}_{3}=0$, the neutrino-electron scattering is

$$
d \sigma=\frac{(2 \pi)^{4-9}}{\left|\mathbf{v}_{1}-\mathbf{v}_{2}\right|} \frac{d^{3} \mathbf{p}_{1}^{\prime} d^{3} \mathbf{p}_{2}^{\prime} d^{3} \mathbf{p}_{3}^{\prime}}{2 E_{1} 2 E_{2} 2 E_{3} 2 E_{1}^{\prime} 2 E_{2}^{\prime} 2 E_{3}^{\prime}} \frac{\delta^{4}\left(\sum p_{i}-\sum p_{f}^{\prime}\right)}{V}\left|\left\langle p_{1}^{\prime} p_{2}^{\prime} p_{3}^{\prime}|T| p_{1} p_{2} p_{3}\right\rangle\right|^{2}
$$

\footnotetext{
${ }^{1} A$ may be considered as a boson, which gives the same result.
} 
where we can take $V$ as the atomic volume to which the flux of neutrinos sweep. For $1 / V$, the sum $B$ in Eq. A9 is used. The T-matrix squared in Eq. (9) is summed over the spins

$$
\frac{1}{\left(2 s_{e}+1\right)\left(2 s_{\nu}+1\right)} \sum_{s_{\nu}, s_{e}} \sum_{s_{\nu}^{\prime}, s_{e}^{\prime}}|\mathrm{Amp}|^{2}=\left(|T|_{\mathrm{Q}}^{2}\right)
$$

If we consider the MM interaction also, then we will have the factor $\left(|T|_{\mathrm{MM}}^{2}+|T|_{\mathrm{Q}}^{2}\right)$ instead of $\left(|T|_{\mathrm{Q}}^{2}\right)$. For the volume $V$, we use the Xenon volume for each principal quantum number $n$ separately. The $n$-th shell volume is $V=(4 \pi / 3)\left(n a_{Z}\right)^{3}$ where $a_{Z}$ is the K-shell radius of Xenon atom. Then, for the $\nu_{\alpha}\left(k_{\nu}\right)+e\left(p_{e}\right)+A\left(P_{A}\right) \rightarrow \nu_{\beta}^{\prime}\left(k_{\nu}^{\prime}\right)+e^{\prime}\left(p_{e}^{\prime}\right)+A^{\prime}\left(P_{A}^{\prime}\right)$ scattering, Eq. (9) becomes

$$
d \sigma=\frac{3 Z^{3} \alpha_{\mathrm{em}}^{3}}{2^{12} \pi^{4}} \frac{m_{e}^{2}\left|\mathbf{p}_{e}^{\prime}\right|^{2} d\left|\mathbf{p}_{e}^{\prime}\right| E_{\nu}^{\prime} d E_{\nu}^{\prime}}{\left(m_{e}+T_{e}\right) E_{\nu} M_{A}^{2}} \delta\left(E_{\nu}+m_{e}-\delta_{B}+M_{A}-E_{\nu}^{\prime}-\left(m_{e}+T_{e}\right)-M_{A}\right)\left|\left\langle p_{1}^{\prime} p_{2}^{\prime} p_{3}^{\prime}|T| p_{1} p_{2} p_{3}\right\rangle\right|^{2} x
$$

where $\delta_{B}$ is the binding energy and $T_{e}$ is the kinetic energy of the final electron $e^{\prime}$, and $x$ is given in Appendix A for the process in consideration. The following scalar products will be useful,

$$
\begin{aligned}
& q^{2}=-2 E_{\nu} E_{\nu}^{\prime}(1-\cos \theta), q=k_{\nu}-k_{\nu}^{\prime}, \\
& k_{\nu} \cdot k_{\nu}^{\prime}=-k_{\nu} \cdot q=k_{\nu}^{\prime} \cdot q=-\frac{q^{2}}{2} \\
& p_{e} \cdot p_{e}^{\prime}=m_{e}^{2}-\frac{q^{2}}{2} \text { assuming atom at rest, } \\
& k_{\nu}^{\prime} \cdot p_{e}=m_{e} E_{\nu}^{\prime}=k_{\nu} \cdot p_{e}^{\prime}=E_{\nu} E_{e}^{\prime}-E_{\nu}\left|\mathbf{p}_{e}^{\prime}\right| \cos \theta_{e},
\end{aligned}
$$

where we used massless neutrinos, $\theta_{e}$ is the polar angle of the outgoing electron direction relative to the incoming neutrino direction, ${ }^{2}$ and the non-relativistic approximation $E_{e}^{\prime} \simeq m_{e}+\frac{\left|\mathbf{p}_{e}^{\prime}\right|^{2}}{2 m_{e}}=m_{e}+T_{e}$ is used.

Firstly, let us show the inverse- $T_{e}$ rule, used in Sec. II. Note the kinematic variables for $\nu_{e}\left(k_{\nu}\right)+e\left(p_{e}\right) \rightarrow \nu_{e}^{\prime}\left(k_{\nu}^{\prime}\right)+$ $e^{\prime}\left(p_{e}^{\prime}\right)$, for (almost) massless neutrinos and in the limit $M_{A}=\infty$,

$$
\begin{aligned}
& E_{\nu}^{\prime}=\left|\mathbf{k}_{\nu}^{\prime}\right|=\sqrt{E_{e}^{\prime 2}+E_{\nu}^{2}-m_{e}^{2}} \\
& 2 k_{\nu} \cdot k_{\nu}^{\prime}=2 E_{\nu} E_{\nu}^{\prime}(1-\cos \theta)
\end{aligned}
$$

where $\theta$ is the angle of the outgoing netrino relative to the incoming neutrino. It is possible to express $\theta$ in terms of $E_{e}^{\prime}\left[19, \cos \theta=\left(E_{\nu}+m_{e}\right) E_{\nu}^{-1} T_{e}^{1 / 2}\left(T_{e}+2 m_{e}\right)^{-1 / 2}\right.$ such that $\left|\mathbf{p}_{\nu}^{\prime}\right| \simeq E_{\nu}^{\prime}$ becomes

$$
E_{\nu}^{\prime} \simeq \sqrt{E_{\nu}^{2}+2 m_{e} T_{e}-2 E_{\nu} \sqrt{2 m_{e} T_{e}}\left(E_{\nu}+m_{e}\right) E_{\nu}^{-1} T_{e}^{1 / 2}\left(T_{e}+2 m_{e}\right)^{-1 / 2}} .
$$

Now, the $\left|\mathbf{k}_{\nu}^{\prime}\right| d\left|\mathbf{k}_{\nu}^{\prime}\right|=E_{\nu}^{\prime} d E_{\nu}^{\prime}=-E_{\nu}^{\prime} d T_{e}$ integration with the energy conservation delta function $\delta\left(f\left(T_{e}\right)\right)$, with the approximation $T_{e} \ll m_{e}$ and $E_{\nu}^{\prime} \simeq E_{\nu}$ in the final formula, we obtain

$$
\frac{d \sigma}{d T_{e}} \simeq \frac{1}{2^{5} \pi m_{e}^{2} T_{e}}
$$

The reason for the $1 / T_{e}$ dependence is the specific $T_{e}$ powers in Eq. 14, and if it is slightly violated then the $1 / T_{e}$ dependence would not result. Thus, we anticipate the inverse- $T_{e}$ rule is not applicable if the momentum carried by the scattered Xenon atom is considered.

Let us now proceed the three-body scattering given in Eq. A14). The four momenta delta function

$$
\delta^{(3)}\left(\mathbf{k}_{\nu}-\mathbf{k}_{\nu}^{\prime}-\mathbf{p}_{e}^{\prime}-\mathbf{P}_{A}^{\prime}\right) \delta\left(E_{\nu}-E_{\nu}^{\prime}-\Delta-\frac{\left|\mathbf{p}_{e}^{\prime}\right|^{2}}{2 m_{e}}-\frac{\left|\mathbf{P}_{A}^{\prime}\right|^{2}}{2 M_{A}}\right)
$$

where $\mathbf{p}_{e}^{\prime}$ and $\mathbf{P}_{A}^{\prime}$ are the momenta of the outing $e^{\prime}$ and outgoing atom $A^{\prime}$ with $m_{e}$ and $M_{A}$, respectively, and $E_{\nu}-E_{\nu}^{\prime}=\Delta=T_{e}+\delta_{B}$ with the binding energy $\delta_{B}$ of the electron in the atom. The energy transfer to the atom is

\footnotetext{
2 The polar angle made by the outgoing neutrino direction relative to the incoming neutrino direction will be denoted as $\theta$.
} 
neglected in the energy-conservation $\delta$ function, since it is so small. The averaged spin sums of $|T|^{2}$ from Eq. 4 is

$$
\begin{aligned}
& \frac{G_{F}^{2}}{2 M_{\mathrm{eff}}^{6}} \operatorname{Tr} \gamma^{0}\left(1+\gamma_{5}\right) \gamma^{\rho} \gamma^{0} \not \not^{\prime} \gamma^{\alpha}\left(1+\gamma_{5}\right) \not k \cdot \operatorname{Tr} \gamma^{0}\left(g_{V}^{*}+g_{A}^{*} \gamma_{5}\right) \gamma_{\rho} \gamma_{0}\left(\not p^{\prime}+m_{e}\right) \gamma^{0} \gamma_{\alpha}\left(g_{V}+g_{A} \gamma_{5}\right)\left(\not p+m_{e}\right) \cdot \operatorname{Tr}\left(\not p^{\prime}+M_{A}\right)\left(\not p+M_{A}\right) \\
& =\frac{G_{F}^{2}}{2 M_{\mathrm{eff}}^{6}} \operatorname{Tr} 2\left(1-\gamma_{5}\right) \gamma^{0} \gamma^{\rho} \gamma^{0} \not k^{\prime} \gamma^{\alpha} \not \not \cdot \operatorname{Tr} \gamma^{0} \gamma_{\rho} \gamma^{0}\left(\not p^{\prime}+m_{e}\right) \gamma_{\alpha} \\
& \quad\left[\left(\left|g_{V}\right|^{2}+\left|g_{A}\right|^{2}\right) \not p-\left(g_{V} g_{A}^{*}+g_{V}^{*} g_{A}\right) \not p \gamma_{5}+\left(\left|g_{V}\right|^{2}-\left|g_{A}\right|^{2}\right) m_{e}-\left(g_{V} g_{A}^{*}-g_{V}^{*} g_{A}\right) m_{e} \gamma_{5}\right] \cdot\left(8 M_{A}^{2}\right) \\
& =\frac{G_{F}^{2}}{2 M_{\mathrm{eff}}^{6}} 2 \cdot 4\left(k^{\prime \rho} k^{\alpha}-k \cdot k^{\prime} g^{\rho \alpha}+k^{\prime \alpha} k^{\rho}-i \varepsilon^{\rho \mu \alpha \nu} k_{\mu}^{\prime} k_{\nu}\right) \times 4\left(\left(\left|g_{V}\right|^{2}+\left|g_{A}\right|^{2}\right)\left(p_{\rho}^{\prime} p_{\alpha}-p \cdot p^{\prime} g_{\rho \alpha}+p_{\alpha}^{\prime} p_{\rho}\right)\right. \\
& \left.\quad+\left(\left|g_{V}\right|^{2}-\left|g_{A}\right|^{2}\right) m_{e}^{2} g_{\rho \alpha}-i\left(g_{V} g_{A}^{*}+g_{V}^{*} g_{A}\right) \varepsilon_{\rho \kappa \alpha \eta} p^{\prime \kappa} p^{\eta}\right) \cdot\left(8 M_{A}^{2}\right) \\
& =\frac{G_{F}^{2}}{2 M_{\mathrm{eff}}^{6}} 2 \cdot 4\left(4\left(\left|g_{V}\right|^{2}+\left|g_{A}\right|^{2}\right)\left(2 k \cdot p k^{\prime} \cdot p^{\prime}+2 k \cdot p^{\prime} k^{\prime} \cdot p\right)+4\left[\left(\left|g_{V}\right|^{2}-\left|g_{A}\right|^{2}\right) m_{e}^{2}\left(-2 k \cdot k^{\prime}\right)\right.\right. \\
& \left.\left.\quad-2 \operatorname{Re}\left(g_{V} g_{A}^{*}\right)\left(k \cdot p k^{\prime} \cdot p^{\prime}-k \cdot p^{\prime} k^{\prime} \cdot p\right)\right]\right) \cdot\left(8 M_{A}^{2}\right) \\
& =\frac{2^{8} G_{F}^{2} M_{A}^{2} m_{e}^{2} E_{\nu}^{2}}{M_{\mathrm{eff}}^{6}}\left(\left(\left|g_{V}\right|^{2}+\left|g_{A}\right|^{2}\right)\left[\left(1-\frac{\left|\mathbf{p}_{e}^{\prime}\right|^{2}}{2 m_{e} E_{\nu}}\right)+\left(\frac{E_{e}^{\prime}}{m_{e}}-\frac{\left|\mathbf{p}_{e}^{\prime}\right|}{m_{e}} \cos \theta_{e}\right)^{2}\right]+\left(\left|g_{V}\right|^{2}-\left|g_{A}\right|^{2}\right) m_{e}^{2}\left(-q^{2} / 2 E_{\nu}^{2}\right)\right. \\
& \left.\quad-\operatorname{Re}\left(g_{V} g_{A}^{*}\right)\left[\left(1-\frac{\left|\mathbf{p}_{e}^{\prime}\right|^{2}}{2 m_{e} E_{\nu}}\right)+\left(\frac{E_{e}^{\prime}}{m_{e}}-\frac{\left|\mathbf{p}_{e}^{\prime}\right|}{m_{e}} \cos \theta_{e}\right)^{2}\right]\right)
\end{aligned}
$$

where we will use $M_{\text {eff }}^{6}$ given in Eq. A11 of Appendix A and $\sum_{s} u_{e_{\text {atom }}}(p) \bar{u}_{e_{\text {atom }}}(p) \simeq \not p+m_{e}$. Here, however, the three momentum conservation is treated accurately. On the other hand, most people use the two body scattering and the uncertaintly here is the probability in finding a prospective bound electron in the atom and the subsequent integration over the probability function of the electron cloud in the atom, which will be very complicated. This calculation is not found in the literature.

\section{B. Magnetic moment contribution $|T|_{\mathrm{MM}}^{2}$}

If neutrinos have magnetic moment, we consider the second term in Eq. (6). In this case, summing over the spins, viz. Eq. (7), we have

$$
\begin{aligned}
|T|_{\mathrm{MM}}^{2} & =\frac{1}{4}\left(\frac{e^{2} f_{M}}{2 M M_{\mathrm{eff}}^{3}}\right)^{2} \frac{q_{\mu} q_{\alpha}}{q^{4}} \operatorname{Tr} \sigma^{\mu \nu}\left(\frac{\not \phi_{i}}{2}\right) \sigma^{\alpha \beta}\left(\frac{\not \phi_{f}}{2}\right) \cdot \operatorname{Tr} \gamma_{\nu}\left(\not p_{i}+m_{e}\right) \gamma_{\beta}\left(\not p_{f}+m_{e}\right) \cdot \operatorname{Tr}\left(\not p^{\prime}+M_{A}\right)\left(\not p+M_{A}\right) \\
& =\frac{1}{64}\left(\frac{e^{2} f_{M}}{2 M M_{\mathrm{eff}}^{3}}\right)^{2} \frac{1}{q^{4}} \operatorname{Tr}\left(\not \gamma^{\nu}-\gamma^{\nu} \not q\right) \not \phi_{i}\left(\not \gamma^{x}-\gamma^{x} \not q\right) \not \phi_{f} \cdot \operatorname{Tr} \gamma_{\nu}\left(\not p_{i}+m_{e}\right) \gamma_{\beta}\left(\not \not p_{f}+m_{e}\right) \cdot\left(8 M_{A}^{2}\right) \\
& =\frac{1}{64}\left(\frac{e^{2} f_{M}}{2 M M_{\mathrm{eff}}^{3}}\right)^{2} \frac{1}{q^{4}} Q^{\nu x} L_{\nu x} \cdot\left(8 M_{A}^{2}\right)
\end{aligned}
$$

where we neglected the neutrino mass. We obtain ${ }^{3}$

$$
\begin{aligned}
Q^{\nu x} \equiv & \operatorname{Tr}\left(\not \gamma^{\nu}-\gamma^{\nu} \not\right)\left(\not k_{i}+m_{\nu}\right)\left(\not \gamma^{x}-\gamma^{x} \not\right)\left(\not k_{f}+m_{\delta}\right) \\
= & 4 g^{\nu x}\left(2 q \cdot k_{i} q \cdot k_{f}\right)+4 k_{i} \cdot k_{f}\left(q^{\nu} q^{x}-q^{2} g^{\nu x}\right)+4 q^{2}\left(k_{i}^{\nu} k_{f}^{x}+k_{i}^{x} k_{f}^{\nu}\right) \\
& -4 q^{\nu}\left(k_{f}^{x} q \cdot k_{i}+k_{i}^{x} q \cdot k_{f}\right)-4 q^{x} k_{f}^{\nu} q \cdot k_{i}+4 q^{x} k_{i}^{\nu} q \cdot k_{f}, \\
L_{\nu x} \equiv & 4\left(p_{\nu}^{i} p_{x}^{f}+p_{\nu}^{f} p_{x}^{i}-p^{i} \cdot p^{f} g_{\nu x}+m_{e}^{2} g_{\nu x}\right),
\end{aligned}
$$

\footnotetext{
3 The trace of six gamma matrices, $(1 / 4) \operatorname{Tr} \gamma^{\rho} d \not b \gamma^{\sigma} k d d=g^{\rho \sigma}(a \cdot b c \cdot d-a \cdot c b \cdot d+a \cdot d b \cdot c)+a \cdot c\left(b^{\rho} d^{\sigma}+b^{\sigma} d^{\rho}\right)+b \cdot d\left(a^{\rho} c^{\sigma}+a^{\sigma} c^{\rho}\right)$ $-a \cdot d\left(b^{\rho} c^{\sigma}+b^{\sigma} c^{\rho}\right)-b \cdot c\left(a^{\rho} d^{\sigma}+a^{\sigma} d^{\rho}\right)+a \cdot b\left(-c^{\rho} d^{\sigma}+c^{\sigma} d^{\rho}\right)+c \cdot d\left(a^{\rho} b^{\sigma}-a^{\sigma} b^{\rho}\right)$, is used.
} 
from which we obtain, neglecting $\mathrm{O}\left(m_{e}^{2} / E_{\nu}^{2}\right)$, using Eq. 12,

$$
\begin{aligned}
\frac{1}{q^{4}} Q^{\nu x} L_{\nu x}= & \frac{1}{q^{4}}\left(32 k_{i} \cdot k_{f} q \cdot p_{i} q \cdot p_{f}+16 q^{2}\left(-k_{i} \cdot k_{f} p_{i} \cdot p_{f}+2 k_{i} \cdot p_{i} k_{f} \cdot p_{f}+2 k_{i} \cdot p_{f} k_{f} \cdot p_{i}\right)\right. \\
& -32 q \cdot k_{i}\left(q \cdot p_{i} k_{f} \cdot p_{f}+q \cdot p_{f} k_{f} \cdot p_{i}\right)-32 q \cdot k_{f}\left(q \cdot p_{i} k_{i} \cdot p_{f}+q \cdot p_{f} k_{i} \cdot p_{i}\right) \\
& \left.+16 m_{e}^{2}\left(4 q \cdot k_{i} q \cdot k_{f}-q^{2} k_{i} \cdot k_{f}\right)\right) \\
= & \frac{1}{q^{4}}\left(-4 q^{6}+16 q^{2}\left(k_{i} \cdot p_{i}+k_{f} \cdot p_{i}\right)\left(k_{i} \cdot p_{f}+k_{f} \cdot p_{f}\right)\right) \\
& =4 Q^{2}-\frac{16}{Q^{2}}\left(k_{i} \cdot p_{i}+k_{f} \cdot p_{f}\right)\left(k_{i} \cdot p_{f}+k_{f} \cdot p_{i}\right)=8 E_{\nu} E_{\nu}^{\prime}(1-\cos \theta)-\frac{8 m_{e}^{2}}{(1-\cos \theta)}
\end{aligned}
$$

where $q^{2}=-Q^{2}$, and $|T|_{\mathrm{MM}}^{2}$ is

$$
|T|_{\mathrm{MM}}^{2}=\frac{\pi^{2}}{2}\left(\frac{f_{\alpha \beta}}{m_{e} M_{\mathrm{eff}}^{3}}\right)^{2} \alpha_{\mathrm{em}}^{2}\left(E_{\nu} E_{\nu}^{\prime}(1-\cos \theta)-\frac{m_{e}^{2}}{(1-\cos \theta)}\right)\left(8 M_{A}^{2}\right) .
$$

Using Eq. (11), we obtain the cross section for the incident neutrino $\nu_{\alpha}$ going to $\nu_{\beta}$ with a MM (including transition MM also), $f_{\alpha \beta}$ (in units of the electron Bohr magneton), ${ }^{4}$

$$
\begin{aligned}
\frac{d \sigma}{d T_{e} d \cos \theta} & =\frac{3 Z^{3} \alpha_{\mathrm{em}}^{3} m_{e}^{3} E_{\nu}^{\prime}\left|\mathbf{p}_{e}^{\prime}\right|}{2^{10} \pi^{4} E_{\nu}\left(m_{e}+T_{e}\right)} 4 \pi^{2}\left(\frac{f_{\alpha \beta}}{m_{e} M_{\mathrm{eff}}^{3}}\right)^{2} \alpha_{\mathrm{em}}^{2}\left(E_{\nu} E_{\nu}^{\prime}(1-\cos \theta)-\frac{m_{e}^{2}}{(1-\cos \theta)}\right) \\
& =\frac{3 Z^{3} \alpha_{\mathrm{em}}^{5} m_{e}^{3}}{2^{8} \pi^{2} M_{\mathrm{eff}}^{6}} \frac{\sqrt{2 T_{e} / m_{e}}}{\left(1+T_{e} / m_{e}\right)^{5 / 2}}\left(1-\frac{\delta_{B}}{m_{e}}-\frac{T_{e}}{m_{e}}\right)^{2}\left((1-\cos \theta)-\frac{m_{e}^{2}}{E_{\nu} E_{\nu}^{\prime}(1-\cos \theta)}\right)\left(f_{\alpha \beta}\right)^{2} \\
& =0.883 \times 10^{-5} \mathrm{MeV}^{-3} \frac{\sqrt{2 T_{e} / m_{e}}}{\left(1+T_{e} / m_{e}\right)^{5 / 2}}\left(1-\frac{\delta_{B}}{m_{e}}-\frac{T_{e}}{m_{e}}\right)^{2}\left((1-\cos \theta)-\frac{m_{e}^{2}}{E_{\nu} E_{\nu}^{\prime}(1-\cos \theta)}\right)\left(f_{\alpha \beta}\right)^{2}
\end{aligned}
$$

leading to

$$
\frac{d \sigma}{d T_{e}}=0.883 \times 10^{-5} \mathrm{MeV}^{-3} \frac{\sqrt{2 T_{e} / m_{e}}}{\left(1+T_{e} / m_{e}\right)^{5 / 2}}\left(1-\frac{\delta_{B}}{m_{e}}-\frac{T_{e}}{m_{e}}\right)^{2}\left(2+\frac{2 m_{e}^{2}}{E_{\nu} E_{\nu}^{\prime}} \ln \frac{1}{\delta_{\text {inf }}}\right)\left(f_{\alpha \beta}\right)^{2}
$$

where $\theta_{\min }$ is near 0 is used, i.e. $\left.\cos \theta\right|_{\max }=\frac{1}{2}-\frac{\sqrt{2 m_{e} T_{e}}}{\left\langle E_{\nu}^{\prime}\right\rangle}$, which is integrated over $T_{e}$ and the energy flux of solar $p p$ neutrinos $[18$ to give

$$
\text { For } \mathrm{MM}: \frac{d \sigma}{d T_{e}} \simeq 1.071 \times 10^{-5} \mathrm{MeV}^{-3}(2-8.288) f_{\alpha \beta}^{2} \text {. }
$$

The infrared divergence in the last term of Eq. (22) is cured by the soft photon emission processes 27, and Eq. 24 is used for $T_{e} \geq 2.5 \mathrm{keV}$. Equation 24 gives a number $-6.73 \times 10^{-5} \mathrm{MeV}^{-3} f_{\alpha \beta}^{2}$. Comparing with the almost flat central region $\left(T_{e}=15-20 \mathrm{keV}\right)$ of Fig. 1 (b), we obtain $\left|f_{\alpha \beta}\right| \leq 0.86 \times 10^{-7}$ which is much larger than that $2.8 \times 10^{-11}$ obtained by the Borexino collaboration [13. The huge difference between our estimation and previous ones seems to be from the over estimation of cross sections in the previous analyses, using the inverse $T_{e}$ rule and the coherent scattering.

\section{Contributions from vector and axial-vector charges $|T|_{Q}^{2}$}

The electromagnetic $F_{1}^{\nu}$ form factor of a neutrino is identified from [1]

$$
\begin{aligned}
& \Lambda_{\mu}(q)=f_{Q}\left(q^{2}\right) \gamma_{\mu}-f_{M}\left(q^{2}\right) i \sigma_{\mu \nu} q^{\nu}+f_{E}\left(q^{2}\right) \sigma_{\mu \nu} q^{\nu} \gamma_{5}+f_{A}\left(q^{2}\right)\left(q^{2} \gamma_{\mu}-q_{\mu} q\right) \\
& F_{1}^{\nu}\left(q^{2}\right)=f_{Q}\left(q^{2}\right)=F_{1}^{\nu}(0)+\left.q^{2} \frac{d F_{1}^{\nu}\left(q^{2}\right)}{d q^{2}}\right|_{q^{2}=0}+\cdots
\end{aligned}
$$

\footnotetext{
${ }^{4}$ We use the incoherent cross sector for $\Delta p \gg 2.5 \mathrm{eV}$.
} 
The charge radius is the $q^{2}$ dependent part,

$$
\tilde{r}^{2} \equiv\left\langle r^{2}\right\rangle=\left.6 \frac{d F_{1}^{\nu}\left(q^{2}\right)}{d q^{2}}\right|_{q^{2}=0} .
$$

The elastic cross section includes the interference of the SM weak interaction and the vector and axial vector couplings of neutrinos with

$$
G_{V}=\frac{1}{2}+2 \sin ^{2} \theta_{W}+e^{2}\left(\frac{\tilde{r}^{2} / 6}{\sqrt{2} G_{F}}-\frac{\varepsilon}{\sqrt{2} G_{F} Q^{2}}\right), \text { and } g_{A}=\frac{1}{2},
$$

where $Q^{2}=-q^{2}>0$ for the $t$-channel scattering. However, we consider the 3 -body scattering to take into account the momentum of the recoiling atom. The parameter describing the atomic electromagnetic effects is described in Appendix A in terms of $B$ and $C$. Inserting (17) into (11), we obtain

$$
\begin{aligned}
\frac{d \sigma}{d T_{e}} & =\frac{3 Z^{3} \alpha_{\mathrm{em}}^{3} G_{F}^{2} m_{e}^{4}}{16 \pi^{4}} \cdot \frac{\sqrt{2 m_{e} T_{e}} E_{\nu}\left(E_{\nu}-\delta_{B}-T_{e}\right)}{M_{\mathrm{eff}}^{6}\left(1+T_{e} / m_{e}\right)} \approx 1.22 \times 10^{-23} \cdot \frac{\sqrt{2 m_{e} T_{e}} E_{\nu}\left(E_{\nu}-\delta_{B}-T_{e}\right)}{\left(1+T_{e} / m_{e}\right)} \cdot \frac{1}{M_{\mathrm{eff}}^{6}} \\
& =6.33 \times 10^{-6} \mathrm{MeV}^{-3} \cdot \frac{\left[\frac{E_{\nu}}{m_{e}}\left(\frac{E_{\nu}}{m_{e}}-\frac{\delta_{B}+T_{e}}{m_{e}}\right)\right]}{\left(1+\frac{T_{e}}{m_{e}}\right)^{4}} \sqrt{\frac{2 T_{e}}{m_{e}}}
\end{aligned}
$$

with $B \simeq 1.144 \times 10^{4}$, where the following $|T|_{Q}^{2}$ for the SM value 23 is

$$
|T|_{Q}^{2}=\frac{8 G_{F}^{2} m_{e}^{2}}{M_{\mathrm{eff}}^{2}}\left\{\left(G_{V}+g_{A}\right)^{2} E_{\nu}^{2}+\left(G_{V}-g_{A}\right)^{2} E_{\nu} E_{\nu}^{\prime}+m_{e} E_{e}^{\prime}\left(g_{A}^{2}-G_{V}^{2}\right)\right\},
$$

and we used $1 / M_{\text {eff }}^{6}$ given in Eq. A11.

\section{Millicharge}

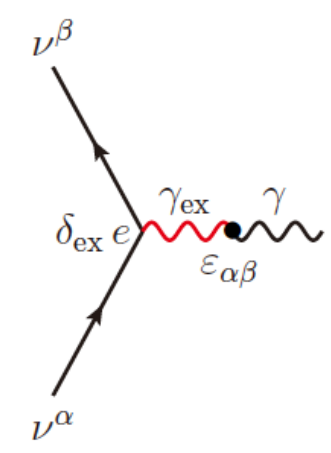

FIG. 2: The neutrino coupling to photon through the kinetic mixing with ex-photon $\gamma_{\mathrm{ex}}$.

There can be another tiny EM property of neutrinos if there exist another (almost) massless U(1) gauge boson. If neutrinos couple to this ex-photon, $\gamma_{\mathrm{ex}}$, neutrinos can carry millicharges via the kinetic mixing [20], for which the Feynman diagram is shown in Fig. 2. Since the SM neutrino is in a SM doublet, L-handed electron $e_{L}$ must carry the same millicharge under the ex-photon gauge group. Then, the magnitude of the $\nu_{\mu}$ coupling to the ex-photon is limited from the $\nu_{\mu}+e$ scattering data (through neutral current coupling by $Z_{\mu}$ ),

$$
\left(\delta_{\text {ex }} e\right)_{\text {from } \nu \text { vertex }} \frac{1}{q^{2}}\left(\delta_{\text {ex }} e\right)_{\text {from e vertex }} \leq \frac{1.167 \times 10^{-5}}{\sqrt{2} \mathrm{GeV}^{2}} \varepsilon\left(e_{L}\right)=0.413 \times 10^{-5} / \mathrm{GeV}^{2}
$$

where $\varepsilon\left(e_{L}\right)\left(=\varepsilon\left(\nu_{L}\right)\right)$ is the neutral current coupling of $e_{L}$ to the $Z$ boson in the SM 22, i.e. 0.5. Since there is no way to pinpoint $\delta_{\mathrm{ex}}$, we set it as 1 , transfering its uncertainty to the kinetic mixing value $\varepsilon=F_{1}^{\nu}(0)$. 


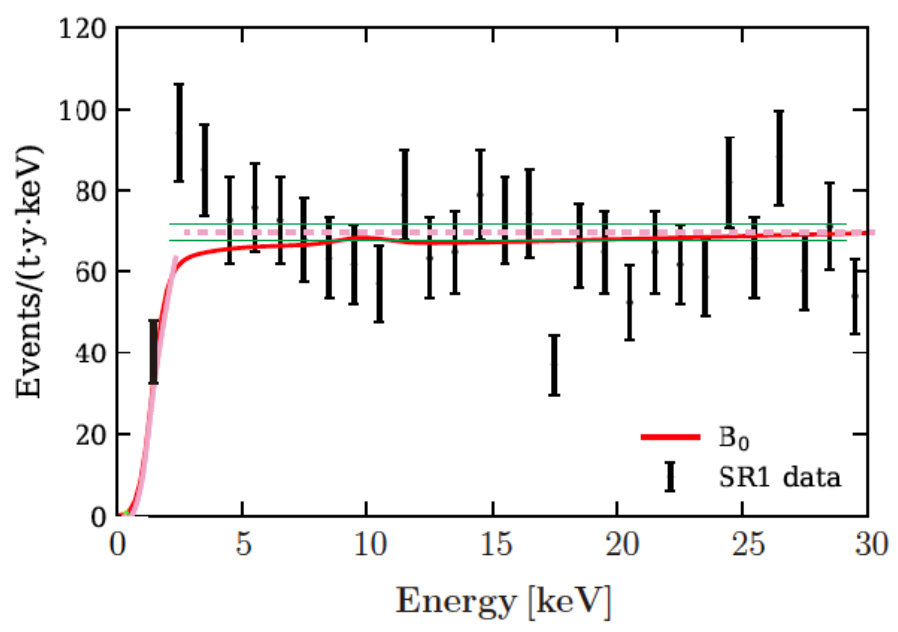

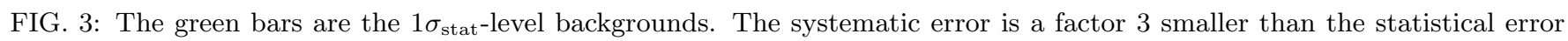
28 .

\section{FITTING}

The contributions from the MM and CR of neutrinos add incoherently. For the MM contribution, we obtained already Eq. (23). For the CR contribution, we should consider the interference with the weak amplitude in the $\mathrm{SM}$, which is given in Eq. 28). From Fig. 1 (b), the cross section is less than $5 \times 10^{-19} \mathrm{MeV}^{-3}$ for $T_{e}>17 \mathrm{keV}$. For $\sin ^{2} \theta_{W} \simeq 0.231$ [22, 24], Eq. 28] gives, with $\langle E\rangle=0.2668$ and $\left\langle E^{2}\right\rangle=0.0788 \mathrm{MeV}^{2}$ of solar $p p$ neutrinos, at $\tilde{r}^{2}=\varepsilon=0$

$$
\text { For } \begin{aligned}
\mathrm{CR}: \simeq & 6.33 \times 10^{-6} \mathrm{MeV}^{-3} \cdot\left\{\left(0.3018-1.02 T_{e, \mathrm{MeV}}\right)[1.462+\Gamma]^{2}\right. \\
& \left.+\left(0.3018-1.02 T_{e, \mathrm{MeV}}\right)[0.462+\Gamma]^{2}\right\} \frac{2.768 T_{e, \mathrm{MeV}}^{1 / 2}}{\left(1+1.957 T_{e, \mathrm{MeV}}\right)^{4}}
\end{aligned}
$$

where $T_{e, \mathrm{MeV}}=T_{e} / \mathrm{MeV}$, which becomes at $T_{e}=17 \mathrm{keV}$

$$
0.5703 \times 10^{-6} \mathrm{MeV}^{-3} \cdot\left\{[1.462+\Gamma]^{2}+[0.462+\Gamma]^{2}\right\} \rightarrow 1.341 \times 10^{-6} \mathrm{MeV}^{-3} \text { for } \Gamma=0 .
$$

The ratio of $5 \times 10^{-19} \mathrm{MeV}^{-3}$ and $(32)$ is $\approx 0.790 \times 10^{-13}$. For solar neutrino energies of order $400 \mathrm{keV}, Q^{2}$ given in 12 is of order $(0.3-0.4 \mathrm{MeV})^{2}$. Taking $\left\langle Q^{2}\right\rangle \simeq 0.1 \mathrm{MeV}^{2}$, the charge radius limit from Eq. $(26)$ is $2.18 \times 10^{-6} \mathrm{MeV}^{-1}$, or $4.30 \times 10^{-17} \mathrm{~cm}$. Note that $\Gamma$ in Eq. 31 is

$$
\begin{aligned}
\Gamma & =4 \pi \alpha_{\mathrm{em}}\left(\frac{\tilde{r}^{2} / 6}{\sqrt{2} G_{F}}-\frac{\varepsilon}{\sqrt{2} G_{F} Q^{2}}\right)=0.9265 \times 10^{9}\left(\tilde{r}^{2} \mathrm{MeV}^{2}-6 \varepsilon_{\mathrm{MeV}}\right) \\
& =2.387 \times 10^{30}\left(\tilde{r}^{2} \mathrm{~cm}^{-2}-2.329 \times 10^{-21} \varepsilon_{\mathrm{MeV}}\right)
\end{aligned}
$$

with $\varepsilon_{\mathrm{MeV}}=\varepsilon / Q_{\mathrm{MeV}}^{2}$ (where $Q_{\mathrm{MeV}}$ is $Q$ in units of $\left.\mathrm{MeV}\right)$.

If we allow millicharges, the $1 \sigma$ error (taken as the \pm 2 events out of 76 in Fig. 11(a)) is shown as the yellow band in the $\tilde{r}^{2}-\varepsilon$ plane of Fig. 4. There already exist bounds on the millicharges of light dark matter from the stellar evolution data, viz. Fig. 12 of [29] and Fig. 1 of [30], presenting $\varepsilon<10^{-12}$ in the dark matter mass range less than $10^{4} \mathrm{eV}$, which however cannot be used for our SM neutrinos. The difference is that we studied in this paper the (almost) massless SM neutrinos which cannot be dark matter in the Universe and we considered the EM properties of neutrinos only from solar neutrinos.

\section{CONCLUSION}

We obtained the bounds on the electromagnetic properties of neutrinos implied by the XENON1T data: a bound on the magnetic moment $\left|f_{\alpha \beta}\right| \leq 0.86 \times 10^{-7}$ (times the electron Bohr magneton), the charge radius $|\tilde{r}|<4.30 \times 10^{-17}$ cm, 


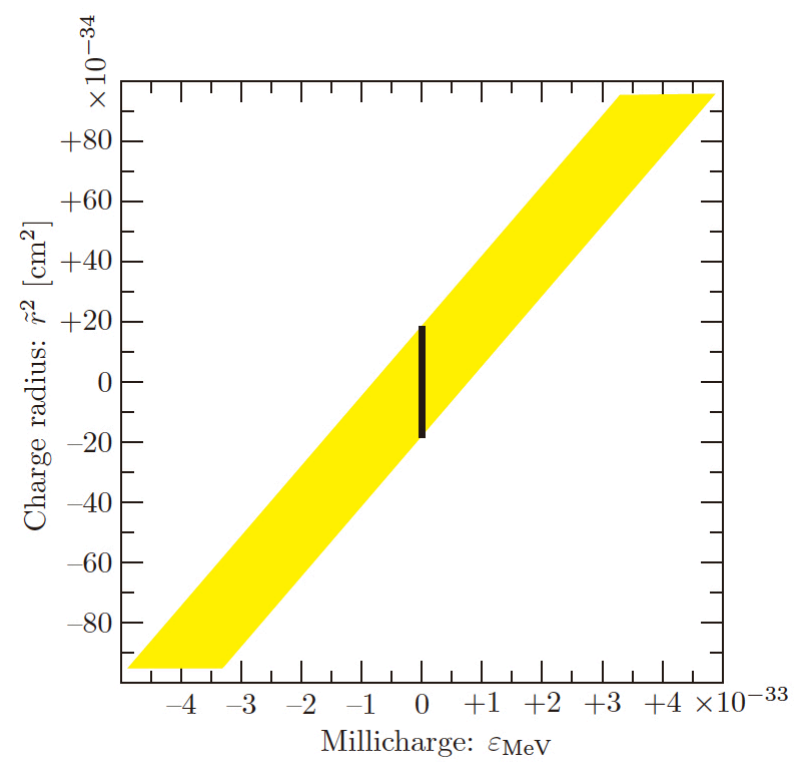

FIG. 4: The bounds on the charge radius (vertical bar) at $\varepsilon=0$ and the $1 \sigma$ yellow band in the charge radius vs. millicharge plane, where $\varepsilon_{\mathrm{MeV}}=\varepsilon / Q_{\mathrm{MeV}}^{2}$ (where $Q_{\mathrm{MeV}}$ is $Q$ in units of $\mathrm{MeV}$ ) depends on the effective momentum transfer in the process.

and the millicharge bound (Fig. 44) if there exists a massless extra photon.

\section{Appendix A: Estimate of probability amplitude}

If an electron is kicked out from an atomic orbit, an electron in the outer orbit will fill that vacancy immediately. The transition is occurring electromagnetically via the $E 1$ or $M 1$ transition. The the $E 1$ transition rate between the eigenstates $|k\rangle$ and $|n\rangle$ is presented a long time ago [31, 32.

$$
\frac{(2 \pi)^{2} \alpha_{\mathrm{em}}}{3} \omega_{k n}^{3}|\langle k|\mathbf{r}| n\rangle|^{2}
$$

where $\mathbf{r}$ is the coordinate of the EM field. We can estimate $\langle k|\mathbf{r}| n\rangle$ for the ground state $(k=n=1)$ as $a_{B} / Z$, with $a_{B}=0.529 \times 10^{-8} \mathrm{~cm}=268.1 \mathrm{MeV}^{-1}$, and for the excited state $n$ as $n a_{B} / Z$. We use $a_{Z}=14.48 \mathrm{keV}^{-1}$ for $Z=54$. Then, the rest of the matrix element takes into account only the spherical harmonics and gives selection rules between angular momentum eigenfunctions. Now we consider kicking out the electron outside the atom and the selection rule always permits it. So, we will consider $\omega_{n n^{\prime}}=\omega_{n \infty}=\left|E_{n} / \hbar\right|$. Thus, the transition rate to kick out an electron to outside is [32],

$$
\frac{4 \alpha_{\mathrm{em}} k^{3}}{3} \frac{1}{2 j_{i}+1}\left|\left\langle n_{f} j_{f}\|D\| n_{i} j_{i}\right\rangle\right|^{2}
$$

Considering the Hydrogenic radial wave function $C_{n} r^{n-1} e^{-r / n a}$ with $C_{n}=(2 / n)^{2 n+1} / \sqrt{4 \pi a_{Z}^{3}}$ with $a_{Z}=a_{B} / Z$, we can estimate the last factor, between the plane wave and the bound state $|n\rangle,\left|2^{2 n}(n+1) ! a_{Z}^{4} / n^{n-1} \sqrt{\pi a_{Z}^{3} V}\right|^{2}$. Thus, the transition rate to kick out an electron to outside by electromagnetic interactions in the atom is

$$
\frac{4 \alpha_{\mathrm{em}} k^{3}}{3} \frac{a_{Z}^{5}}{\pi V}\left(\frac{2^{2 n+1}[(n+1) !]}{n^{n-1}}\right)^{2} .
$$

Let us introduce a parameter $M_{\text {eff }}$ reproducing Eq. A3, using the following effective interaction

$$
\frac{1}{M_{\mathrm{eff}}^{2}} \bar{e} \gamma^{\mu} e \bar{A} \gamma_{\mu} A
$$


and we use particle physicists' $2 \rightarrow n$ transition rate,

$$
\text { Rate }=(2 \pi)^{4-3 n} \frac{d^{3} \mathbf{p}_{1}^{\prime} \cdots d^{3} \mathbf{p}_{n}^{\prime}}{2 E_{1} 2 E_{2} 2 E_{1}^{\prime} 2 E_{2}^{\prime} \cdots 2 E_{n}^{\prime}} \frac{\delta^{4}\left(p_{1}+p_{2}-p_{1}^{\prime} \cdots-p_{n}^{\prime}\right)}{V}\left|\left\langle p_{1}^{\prime} \cdots p_{n}^{\prime}|T| p_{1} p_{2}\right\rangle\right|^{2} .
$$

The two-body scattering rate from Eq. A5, using (A4) and averaging over a flux of incoming particles on a rest target, is

$$
\begin{aligned}
& (2 \pi)^{-2} \frac{d^{3} \mathbf{p}_{e}^{\prime}}{2^{4} m_{e} E_{e}^{\prime} M_{A}^{2}} \frac{\delta\left(\sum_{i} E_{i}-\sum_{f} E_{f}\right)}{V} \frac{2^{6}}{M_{\mathrm{eff}}^{4}} m_{e} E_{e}^{\prime} M_{A}^{2} \\
& =\frac{4\left|\mathbf{p}_{e}^{\prime}\right|^{2} d\left|\mathbf{p}_{e}^{\prime}\right|}{\delta\left(E_{\nu}^{\prime}+m_{e}+\frac{\left|\mathbf{p}_{e}^{\prime}\right|^{2}}{2 m_{e}}+M_{A}-E_{\nu}-m_{e}+\delta_{B}-M_{A}\right)} \frac{1}{\pi V}=4 \pi^{-1} \frac{m_{e}\left|\mathbf{p}_{e}^{\prime}\right|}{M_{\mathrm{eff}}^{4} V}
\end{aligned}
$$

where we used

$$
\begin{array}{r}
16\left(p_{e}^{\mu} p_{e}^{\prime \nu}-p_{e}^{\prime} \cdot p_{e} g^{\mu \nu}+p_{e}^{\nu} p_{e}^{\prime \mu}+m_{e}^{2} g^{\mu \nu}\right)\left(P_{A}^{\mu} P_{A \nu}^{\prime}-P_{A}^{\prime} \cdot P_{A} g_{\mu \nu}+P_{A}^{\nu} P_{A \mu}^{\prime}+M_{A}^{2} g_{\mu \nu}\right)= \\
16\left(2 p_{e} \cdot P_{A} p_{e}^{\prime} \cdot P_{A}^{\prime}-2 p_{e}^{\prime} \cdot p_{e} P_{A}^{\prime} \cdot P_{A}+2 p_{e} \cdot P_{A}^{\prime} p_{e}^{\prime} \cdot P_{A}+2 m_{e}^{2} P_{A} \cdot P_{A}^{\prime}\right. \\
-\left(P_{A} \cdot P_{A}^{\prime}\right)\left(-2 p_{e} \cdot p_{e}^{\prime}+4 m_{e}^{2}\right)+M_{A}^{2}\left(-2 p_{e} \cdot p_{e}^{\prime}+4 m_{e}^{2}\right) \\
\rightarrow \frac{32}{M_{\mathrm{eff}}^{4}}\left(p_{e} \cdot P_{A} p_{e}^{\prime} \cdot P_{A}^{\prime}+p_{e} \cdot P_{A}^{\prime} p_{e}^{\prime} \cdot P_{A}-M_{A}^{2} p_{e} \cdot p_{e}^{\prime}+m_{e}^{2} M_{A}^{2}\right) \\
\simeq \frac{32}{M_{\mathrm{eff}}^{2}}\left(3 m_{e}^{2} M_{A}^{2}-m_{e} E_{e}^{\prime} M_{A}^{2}\right) \simeq \frac{2^{6}}{M_{\mathrm{eff}}^{4}} m_{e} E_{e}^{\prime} M_{A}^{2} .
\end{array}
$$

This can be compared to A3, and we obtain

$$
\frac{1}{M_{\mathrm{eff}}^{2}}=\sqrt{\frac{\alpha_{\mathrm{em}}}{3}} \frac{a_{Z}^{5 / 2}}{\sqrt{m_{e}\left(m_{e}+T_{e}\right)}} k_{n}^{3 / 2}\left(\frac{2^{2 n+1}[(n+1) !]}{n^{n-1}}\right),
$$

where we use $k_{n}$ for the threshold value, $k_{n} \simeq\left|E_{n}(X e)\right| / \hbar$. In practice, let us use Eq. A3 summing over the electrons from each orbit of the Xenon atom with $k_{n}^{3}$,

$$
\begin{aligned}
& A \equiv \sum_{n=1}^{5} \frac{1}{n^{3}} f(n)\left(\frac{2^{2 n+1}[(n+1) !]}{n^{n-1}}\right)^{2}=7.028 \times 10^{5}, \\
& B \equiv \sum_{n=1}^{5} \frac{1}{n^{6}} f(n)\left(\frac{2^{2 n+1}[(n+1) !]}{n^{n-1}}\right)^{2} \simeq 1.144 \times 10^{4},
\end{aligned}
$$

where $f(n)=2,8,18,18,8$ for $n=1,2, \cdots, 5$, respectively. This takes into account the number of electrons in the Xenon atom $Z$, corresponding to the incoherent process. $B$ appears when we takes into account $V$ in the denominator, which is the case in the 3-body scattering. Thus, we estimate, for the Xenon atom of $Z=54$ and $M_{A} \simeq 7.028 \times 10^{5} \mathrm{MeV}$

$$
\frac{1}{M_{\mathrm{eff}}^{2}}=\sqrt{\frac{\alpha_{\mathrm{em}}}{3}} \frac{\sqrt{A}}{Z^{5 / 2} m_{e}^{2} \sqrt{1+T_{e} / m_{e}}}=0.150\left(1+T_{e} / m_{e}\right)^{-1 / 2} \mathrm{MeV}^{-2}
$$

If we use A10, then

$$
\frac{1}{M_{\mathrm{eff}}^{6}}=5.69 \times 10^{4} \frac{\mathrm{keV}^{-15 / 2}}{\left[m_{e}\left(m_{e}+T_{e}\right)\right]^{3 / 2}} \sum_{n} k_{n}^{9 / 2}\left(\frac{2^{2 n+1}[(n+1) !]}{n^{n-1}}\right)^{3} \equiv 2.27 \times 10^{5} \frac{\mathrm{MeV}^{-3}}{\left[m_{e}\left(m_{e}+T_{e}\right)\right]^{3 / 2}} C
$$

where $C$ becomes $^{5}$

$$
C \simeq 2.287 \times 10^{12}
$$

\footnotetext{
${ }^{5}$ If we use $Z_{\text {eff }}(n)$, including electron interactions, we obtain $C=5.136 \times 10^{12}$, and $1 / M_{\mathrm{eff}}^{6}=1.17 \times 10^{18}\left[m_{e}\left(m_{e}+T_{e}\right)\right]^{-3 / 2} \mathrm{MeV}^{-3}$.
} 
The $3 \rightarrow n$ transition rate is

$$
\text { Rate }=(2 \pi)^{4-3 n} \frac{d^{3} \mathbf{p}_{1}^{\prime} \cdots d^{3} \mathbf{p}_{n}^{\prime}}{2 E_{1} 2 E_{2} 2 E_{3} 2 E_{1}^{\prime} 2 E_{2}^{\prime} \cdots 2 E_{n}^{\prime}} \frac{\delta^{4}\left(p_{1}+p_{2}+p_{3}-p_{1}^{\prime} \cdots-p_{n}^{\prime}\right)}{V^{2}}\left|\left\langle p_{1}^{\prime} \cdots p_{n}^{\prime}|T| p_{1} p_{2} p_{3}\right\rangle\right|^{2}
$$

from which we calculate the three body scattering cross section. A flux of incoming particles 1 (neutrino in our case) scatter on two particles 2 (electron in our case) and 3 (atom in our case). Thus, the cross section consists of two parts, one with a flux factor $1 /\left|\mathbf{v}_{1}-\mathbf{v}_{2}\right|$ and the other $1 /\left|\mathbf{v}_{1}-\mathbf{v}_{3}\right|$. Taking $\mathbf{v}_{3}=0$, the neutrino-electron scattering is

$$
d \sigma=\frac{(2 \pi)^{4-9}}{\left|\mathbf{v}_{1}-\mathbf{v}_{2}\right|} \frac{d^{3} \mathbf{p}_{1}^{\prime} d^{3} \mathbf{p}_{2}^{\prime} d^{3} \mathbf{p}_{3}^{\prime}}{2 E_{1} 2 E_{2} 2 E_{3} 2 E_{1}^{\prime} 2 E_{2}^{\prime} 2 E_{3}^{\prime}} \frac{\delta^{4}\left(\sum p_{i}-\sum p_{f}^{\prime}\right)}{V}\left|\left\langle p_{1}^{\prime} p_{2}^{\prime} p_{3}^{\prime}|T| p_{1} p_{2} p_{3}\right\rangle\right|^{2}
$$

where we can take $V$ as the atomic volume to which the flux of neutrinos sweep. For $1 / V$, the sum $B$ in Eq. A9 will be used below. The $T$-matrix squared in Eq. A14 is summed over the spins

$$
\frac{1}{\left(2 s_{e}+1\right)\left(2 s_{\nu}+1\right)} \sum_{s_{\nu}, s_{e}} \sum_{s_{\nu}^{\prime}, s_{e}^{\prime}}|\mathrm{Amp}|^{2}=\left(|T|_{\mathrm{Q}}^{2}\right)
$$

If we consider the MM interaction also, then we will have a factor $\left(|T|_{\mathrm{MM}}^{2}+|T|_{\mathrm{Q}}^{2}\right)$ instead of $\left(|T|_{\mathrm{Q}}^{2}\right)$. For the volume $V$, we use the Xenon volume for each $n$ separately. The $n$-th shell volume we used $V=(4 \pi / 3)\left(n a_{Z}\right)^{3}$.

\section{Acknowledgments}

We thank Kyungwhan Ahn, Elena Aprile, Ki-Young Choi, Paul Frampton, Wonho Jhe, Junu Jeong, Sin-Kyu Kang, Sejin Kim, and Myungbo Shim for useful discussions. J.E.K. thanks the APCTP, for the hospitality extended to him during his visit, where this work has been started. J.E.K. is supported in part by the National Research Foundation (NRF) grant NRF-2018R1A2A3074631, and S.Y. is supported in part by the Institute for Basic Science (IBS-R017D1-2020-a00/IBS-R017-Y1-2020- 345 a00).

[1] W. Pauli, Letter to the Tübingen meeting of the German Physical Society, December 4, 1930.

[2] F. Reines and C. L. Cowan, Neutrino magnetic moment upper limit, Phys. Rev. 107, 528 (1957) [doi:10.1103/PhysRev.107.528].

[3] J. Bernstein and T. D. Lee, Electromagnetic form factor of the neutrinos, Phys. Rev. Lett. 11, 512 (1963) [doi:10.1103/PhysRevLett.11.512].

[4] R. B. Clark and R. D. Pedigo, Forward-peaked $\nu_{e}$ e scattering and the solar-neutrino problem, Phys. Rev. D 8, 2261 (1973) [doi: 10.1103/PhysRevD.8.2261].

[5] J. E. Kim, V. S. Mathur, and S. Okubo, Electromagnetic properties of the neutrino from neutral-current experiments, Phys. Rev. D 9, 3050 (1974) [doi: 10.1103/PhysRevD.9.3050].

[6] J. E. Kim, Effects of the transition magnetic moment of the neutrino, Phys. Rev. Lett. 41, 360 (1978) [doi: 10.1103/PhysRevLett.41.360].

[7] L. B. Okun, M. B. Voloshin, and M. Y. Vysotsky, Sov. J. Nucl. Phys. 44 (1986) 440, Yad. Fiz. 44 (1986) $677-680$ [Sov. Phys. JETP 64 (1986) 446-452], and Zh. Eksp. Teor. Fiz. 91 (1986) 754-765.

[8] R. Barbieri, R. N. Mohapatra and T. Yanagida, The magnetic moment of the neutrino and its implications for neutrino signal from SN1987a, Phys. Lett. B 213, 69 (1988) [doi: 10.1016/0370-2693(88)91049-0].

[9] J. E. Kim, Neutrino magnetic moment, Phys. Rev. D 14, 3000 (1976) [doi: 10.1103/PhysRevD.14.3000].

[10] M. Fukugita and T. Yanagida, Neutrino oscillation in matter induced by charged scalar particles, Phys. Lett. B 206, 93 (1988) [doi: 10.1016/0370-2693(88)91268-3].

[11] See, for example, C. Giunti and A. Studenikin, Neutrino electromagnetic interactions: A window to new physics, Rev. Mod. Phys. 87, 531 (2015) [e-Print: 1403.6344 [hep-ph]].

[12] For a review, see, J. E. Kim, History of neutrino magnetic moment, e-Print: 1911.06883 [hep-ph].

[13] Borexino Collaboration (M. Agostini et al.), Limiting neutrino magnetic moments with Borexino Phase-II Solar neutrino data, Phys. Rev. D 96, 091103 (2017) [e-Print: 1707.09355 [hep-ex]].

[14] TEXONO Collaboration (M. Deniz et al.), Measurement of $\bar{\nu}_{e}$-electron scattering cross-section with a CsI(Tl) scintillating crystal array at the Kuo-Sheng nuclear power reactor, Phys. Rev. D 81, 072001 (2010) [e-Print: 0911.1597 [hep-ex]].

[15] K. Fujikawa and R. Shrock, The magnetic moment of a massive neutrino and neutrino spin rotation, Phys. Rev. Lett. 45, 963 (1980) [doi: 10.1103/PhysRevLett.45.963].

[16] J. Baumann, R. Gaehler, J. Kalus, and W. Mampe, Experimental limit for the charge of the free neutron, Phys. Rev. D 37, 3107 (1988) [doi: 10.1103/PhysRevD.37.3107]. 
[17] E. Aprile et al.(XENON Collaboration), Excess electronic recoil events in XENON1T, Phys. Rev. D 102, 072004 (2020) [e-print:2006.097121 [hep-ex]].

[18] J. N. Bahcall and C. Peña-Garay, Solar models and solar neutrino oscillations, New J. Phys. 6, 63 (2004).

[19] See, for example, P. Vogel and J. Engel, Neutrino electromagnetic form factors, Phys. Rev. D 39, 3378 (1989) [doi: 10.1103/PhysRevD.39.3378].

[20] B. Holdom, Two U(1)s and $\varepsilon$ charge shift, Phys. Lett. B 166, 196 (1986).

[21] For more references, see, J.-H. Huh, J. E. Kim, J.-C. Park, and S. C. Park, Galactic 511 keV line from MeV milli-charged dark matter, Phys. Rev. D 77, 123503 (2007) [eprint: 0711.3528 [astro-ph]].

[22] J. E. Kim, P. Langacker, M. Levine, and H. H. Williams, A theoretical and experimental review of the weak neutral current: A determination of its structure and limits on deviations from the minimal $S U(2)_{L} \times U(1)$ electroweak theory, Rev. Mod. Phys. 53, 211 (1981) [doi:10.1103/RevModPhys.53.211].

[23] G. 't Hooft, Prediction for neutrino-electron cross-sections in Weinberg's model of weak interactions, Phys. Lett. B 37, 195 (1971) [doi:10.1016/0370-2693(71)90050-5].

[24] A. Bodek, Measurement of the effective weak mixing angle $\sin ^{2} \theta_{\text {eff }}^{\text {lept }}$ from the forward-backward asymmetry of Drell-Yan events at CMS, [eprint:1808.03170 [hep-ex]]. It has not changed much from the value of 1980's 22.

[25] W. S. Cho, J.-H. Huh, I.-W. Kim, J. E. Kim, and B. Kyae, Constraining WIMP magnetic moment from CDMS II experiment, Phys. Lett. B 687, 6 (2010) and 694, 496 (E) (2011) [e-Print: 1001.0579 [hep-ph]].

[26] J. D. Vergados, Searching for light WIMPs via their interaction with electrons, J. Phys. G47, 095007 (2020) [eprint:1911.12956 [hep-ph]].

[27] F. Bloch and A. Nordsieck, Note on the Radiation Field of the Electron, Phys. Rev. 52, 54 (1937) [doi:10.1103/PhysRev.52.54].

[28] E. Aprile, a private communication.

[29] W. L. Xu, C. Dvorkin, and A. Chael, Probing sub-GeV dark matter-baryon scattering with cosmological observables, Phys. Rev. D 97, 103530 (2018) [eprint: 1802.06788 [astro-ph.CO]].

[30] E. D. Kovetz, V. Poulin, V. Gluscevic, K. K. Boddy, R. Barkana, and M. Kamionkowski, Tighter limits on dark matter explanations of the anomalous EDGES 21cm signal, Phys. Rev. D 98, 103529 (2018) [eprint: 1807.11482 [astro-ph.CO]].

[31] L. I. Schiff, Quantum Mechanics (McGraw-Hill Book Co., New York, 1955) p.403, Eq. (45.22).

[32] K. Gottfried and T-M. Yan, Quantum Mechanics: Fundamentals (Springer, Heidelberg, Germany, 2003$)$, p.466.

[33] E. Vitagliano, J. Redondo, and G. Raffelt, Solar neutrino flux at keV energies, JCAP 12, 010 (2017) [e-print: 1708.02248 [hep-ph]]. 